PROCEEDINGS OF THE

AMERICAN MATHEMATICAL SOCIETY

Volume 125, Number 5, May 1997, Pages 1399-1405

S 0002-9939(97)03719-2

\title{
STABILITY IN THE GROMOV-SHUBIN INDEX THEOREM
}

\author{
LIH-CHUNG WANG
}

(Communicated by Palle E. T. Jorgensen)

\begin{abstract}
We investigate the upper semicontinuity of the dimension of the solution space for an elliptic equation with conditions on a compact nowhere dense set.
\end{abstract}

\section{INTRODUCTION}

The Riemann-Roch theorem on a compact Riemann surface can be considered as a connection between the dimension of a space of meromorphic functions and the dimension of a space of meromorphic $(1,0)$-forms. The notion of 'divisor', which is a finite set in the given Riemann surface with an integer (multiplicity) assigned to each point in this finite set, is introduced to study the information about poles and zeros. The result (the difference of the two dimensions) involves the degree of the divisor, which is the sum of all multiplicities.

Gromov and Shubin [G-S] proved a generalization of the classical Riemann-Roch theorem for solutions of general elliptic equations with singularities supported on compact nowhere dense sets. They introduced a notion of 'rigged divisor' which includes two disjoint nowhere dense compact sets with finite-dimensional distribution spaces supported on them. The singularities on the first given set are described as singularities of solutions which may be extended as distributions to the whole given manifold so that after appplying the given elliptic operator we reach the first given space of distributions. The conditions imposed on the second compact set are orthgonality conditions to the second space of distributions. Their main result connects the dimension of the space of solutions having the allowed singularities and satisfying the imposed conditions, with another dimension defined in the same way from the inverse divisor which is obtained by changing places of two given compact subsets and distribution spaces (and replacing the given operator by the adjoint operator). As in the classical Riemann-Roch theorem the corresponding formula includes a degree of the divisor. The degree is defined in terms of the dimensions of the given distribution spaces and two other naturally arising 'secondary' distribution spaces.

When the rigged divisor moves on the manifold, we know that the index of the elliptic operator is invariant. Hence, the degree of the rigged divisor is invariant. Actually the degree is the difference of the index of a naturally arising operator and

Received by the editors September 19, 1995 and, in revised form, November 14, 1995.

1991 Mathematics Subject Classification. Primary 46E99, 58G10.

Key words and phrases. Distribution, elliptic operator, Fredholm operator, rigged divisor, Sobolev space, index, Riemann-Roch theorem, semicontinuity.

(C) 1997 American Mathematical Society 
the index of the given elliptic operator. We want to study the semicontinuity of the dimensions of these distributional spaces. In order to apply the theory of Fredholm operators, we need to let all these spaces lie naturally in Hilbert spaces. This is obtained below by considering the intersection of the $L^{2}$-Sobolev space and the spaces of distributional sections. Here the Sobolev space has sufficiently negative order of Bessel potential. The semicontinuity of the dimensions of spaces can be shown by the theory of Fredholm operators, which reduces the semicontinuity of the dimensions of the distributional spaces in infinite dimensional space to the semicontinuity of the dimensions of the subspaces in the fibre vector space of a vector bundle.

Section 2 contains necessary definitions and notations. In section 3 the semicontinuity of the dimensions of distributional spaces is proved. Section 4 contains some concrete computation of some examples. For the sake of the completeness, there is an appendix which gives a proof of a theorem about Fredholm operators.

\section{Definitions AND PRELIMINARIES}

Let $E$ be a $C^{\infty}$ complex vector bundle over a compact closed $C^{\infty}$-manifold $X$ and $C^{\infty}(U, E)$ be the linear space of all $C^{\infty}$-sections of $E$ over an open set $U$ of $X$. For any open set $U$ denote by $\mathcal{D}^{\prime}(U, E)$ the space of all distributional sections of $E$ over $U$. If $D$ is a closed subset in $X$, let $\mathcal{E}_{D}^{\prime}(X, E)$ be the linear space of all $f \in \mathcal{D}^{\prime}(X, E)$ such that $\operatorname{supp} f \subset D$. Let $\Omega(X)$ be the density bundle over $X . E^{*}$ denotes an antidual vector bundle which is supplied with a $C^{\infty}$-bilinear or sesquilinear nondegenerate duality of bundles $E \times E^{*} \rightarrow \Omega(X)$.

Let $A$ be an elliptic linear differential operator of order $d$ between vector bundles $E$ and $F$, i.e.,

$$
A: C^{\infty}(X, E) \longrightarrow C^{\infty}(X, F)
$$

and let $A^{*}$ be the adjoint operator of $A$, which is again an elliptic linear differential operator of order $d$,

$$
A^{*}: C^{\infty}\left(X, F^{*}\right) \longrightarrow C^{\infty}\left(X, E^{*}\right)
$$

such that $(A u, v)=\left(u, A^{*} v\right)$, where $u \in C^{\infty}(X, E), v \in C^{\infty}\left(X, F^{*}\right)$.

Let $H_{(s)}(X ; E)$ be the $s$-th order space of $L^{2}$-Sobolev distributional sections. Then $A$ defines a Fredholm operator from $H_{(s)}(X ; E)$ to $H_{(s-d)}(X ; F)$.

Let us define a rigged divisor (associated with $A$ ) to be a tuple

$$
\mu=\left(D^{+}, L^{+} ; D^{-}, L^{-}\right),
$$

where $D^{ \pm}$are compact (closed) nowhere dense disjoint subsets in $X, L^{ \pm}$are finitedimensional linear spaces of distributional sections,

$$
\begin{gathered}
L^{+} \subset \mathcal{E}_{D^{+}}^{\prime}(X, F) \cap H_{(s-d)}(X ; F), \\
L^{-} \subset \mathcal{E}_{D^{-}}^{\prime}\left(X, E^{*}\right) \cap H_{(s-d)}\left(X ; E^{*}\right) .
\end{gathered}
$$

We also need to define the following spaces:

$$
\begin{gathered}
\tilde{L}^{+}=\left\{u \mid u \in \mathcal{E}_{D^{+}}^{\prime}(X, E) \cap H_{(s)}(X ; E), A u \in L^{+}\right\}, \\
\tilde{L}^{-}=\left\{v \mid v \in \mathcal{E}_{D^{-}}^{\prime}\left(X, F^{*}\right) \cap H_{(s)}\left(X ; F^{*}\right), A^{*} v \in L^{-}\right\} .
\end{gathered}
$$

Then we can define the degree of a rigged divisor $\mu$ to be the following integer:

$$
\operatorname{deg}_{A}(\mu)=\left(\operatorname{dim} L^{+}-\operatorname{dim} \tilde{L}^{+}\right)+\left(\operatorname{dim} L^{-}-\operatorname{dim} \tilde{L}^{-}\right) .
$$


Because of the elliptic regularity, $A\left(A^{*}\right)$ is injective on $\mathcal{E}_{D^{+}}^{\prime}(X, E)\left(\mathcal{E}_{D^{-}}^{\prime}\left(X, F^{*}\right)\right)$. Hence $\operatorname{dim} L^{ \pm} \geq \operatorname{dim} \tilde{L}^{ \pm}$.

Define the inverse divisor of a rigged divisor $\mu=\left(D^{+}, L^{+} ; D^{-}, L^{-}\right)$associated with the elliptic operator $A$ to be the rigged divisor

$$
\mu^{-1}=\left(D^{-}, L^{-} ; D^{+}, L^{+}\right)
$$

associated with the adjoint elliptic operator $A^{*}$.

Denote by $L(\mu, A)$ the space of solutions with allowed singularities on $D^{+}$and vanishing conditions on $D^{-}$, i.e.,

$$
\begin{array}{r}
L(\mu, A)=\left\{u \mid u \in C^{\infty}\left(X-D^{+}, E\right), \exists \tilde{u} \in \mathcal{D}^{\prime}(X, E), \tilde{u}=u \text { on } X-D^{+},\right. \\
\left.A \tilde{u} \in L^{+},\left(u, L^{-}\right)=0\right\} .
\end{array}
$$

We denote

$$
r(\mu, A)=\operatorname{dim} L(\mu, A) .
$$

Gromov and Shubin proved the following formula:

\section{Gromov-Shubin-Riemann-Roch theorem.}

$$
r(\mu, A)-r\left(\mu^{-1}, A^{*}\right)=\operatorname{ind} A+\operatorname{deg}_{A}(\mu)
$$

where ind $A=\operatorname{dim} \operatorname{Ker} A-\operatorname{dim}$ Coker $A$ is the index of the elliptic operator $A$.

They also proved a duality theorem. Before stating it, we need to introduce the following spaces:

$$
\begin{array}{r}
\Gamma(X, \mu, A)=\left\{u \mid u \in C^{\infty}\left(X-D^{+}, E\right), \exists \tilde{u} \in \mathcal{D}^{\prime}(X, E), \tilde{u}=u \text { on } X-D^{+},\right. \\
\left.A \tilde{u} \in L^{+}+C^{\infty}(X, F),\left(u, L^{-}\right)=0\right\},
\end{array}
$$

and

$$
\tilde{\Gamma}_{\mu}(X, A)=\left\{f \mid f \in C^{\infty}(X, F),\left(f, \tilde{L}^{-}\right)=0\right\} .
$$

Given a section $u \in \Gamma(X, \mu, A)$, we can extend $A u$ to a $C^{\infty}$-section in $C^{\infty}(X, F)$. Therefore, $\tilde{A}$ defines a map

$$
\tilde{A}: \Gamma(X, \mu, A) \longrightarrow \tilde{\Gamma}_{\mu}(X, A) .
$$

By definition, we obtain

$$
\operatorname{Ker} \tilde{A}=L(\mu, A) .
$$

Then we have the following duality theorem:

Gromov-Shubin Duality. (i) $f \in \operatorname{Img} \tilde{A}$ iff $f \in \tilde{\Gamma}_{\mu}(X, A)$ and $\left(f, \operatorname{Ker} \tilde{A}^{*}\right)=0$.

(ii) $\operatorname{dim}$ Coker $\tilde{A}=\operatorname{dim} \operatorname{Ker} \tilde{A}^{*}$.

By the duality theorem, the above Riemann-Roch theorem is equivalent to the following formula:

$$
\text { ind } \tilde{A}=\operatorname{ind} A+\operatorname{deg}_{A}(\mu) \text {. }
$$

Let us introduce the space

$$
\tilde{L}=\left\{v \mid v \in \mathcal{E}_{D^{-}}^{\prime}\left(X, F^{*}\right) \cap H_{(s)}\left(X ; F^{*}\right), A^{*} v \in L^{-}\right\}
$$

and the space of germs of $C^{\infty}$-sections of $E$ on any subset $D$ :

$$
C^{\infty}(D, E)=\lim _{U \supset D} C^{\infty}(U, E),
$$


where $U$ runs through the set of all open neighborhoods of $D$. By the duality theorem, we can get the following statement:

Local solvability theorem ([G-S]). If $f \in C^{\infty}(D, F)$ and $(f, \tilde{L})=0$, then there exists $u \in C^{\infty}(D, E)$ such that $A u=f$ and $(u, L)=0$.

\section{Semicontinuity theorem}

The semicontinuity of the dimension of the kernel of a map between two vector bundles is well known:

Lemma 1. Let $T(z)$ be a linear map from $E_{z}$ to $F_{z}$, where $E_{z}$ and $F_{z}$ are the fibres of two vector bundles $E$ and $F$ at a point $z$. If $T(z)$ is continuous, then the dimension of $\operatorname{Ker} T(z)$ is upper semicontinuous. Moreover, if $T(z)$ is analytic, then the jumping locus is analytic.

Proof. Because

$$
\operatorname{dim} \operatorname{Ker} T(z)+\operatorname{Rank} T(z)=\operatorname{dim} E_{z},
$$

showing that $\operatorname{dim} \operatorname{Ker} T(z)$ is upper semicontinuous is equivalent to showing that $\operatorname{Rank} T(z)$ is lower semicontinuous. Denote by $M(z)$ the associated matrix of $T(z)$ for some basis of $E_{z}$. Let $F_{k}$ be the closed zero locus of determinants of $k \times k$ minors of $M(z)$. Then $\operatorname{Rank} T(z)$ is the largest $k$ such that $z$ does not belong to $F_{k}$. Hence, $\operatorname{Rank} T(z)$ is lower semicontinuous.

If $T(z)$ is analytic, then $F_{k}$ is an analytic set (zero locus of analytic functions). In particular, the jumping locus is analytic, since the jumping locus is $F_{k}$, where the index $k$ is the generic rank of $T(z)$.

Theorem 2. If the rigged divisor $\mu(z)$ and the given elliptic operator $A(z)$ are continuously parametrized, then $r(\mu(z), A(z))$ is upper semicontinuous.

Proof. By the definitions, we have

$$
r(\mu(z), A(z))=\operatorname{dim} L(\mu(z), A(z))=\operatorname{dim} \operatorname{Ker} \tilde{A}(z),
$$

where $\tilde{A}: \Gamma(X, \mu, A) \longrightarrow \tilde{\Gamma}_{\mu}(X, A)$. (See the previous section for notations.) The dimension of the cokernel of $\tilde{A}$ is also finite because of the duality theorem. Hence, $\tilde{A}$ is a Fredholm operator. By the way, we know that the Sobolev spaces are Hilbert spaces. By the theorem in the appendix, we can reduce the semicontinuity problem of $\operatorname{dim} \operatorname{Ker} \tilde{A}(z)$ for infinite dimensional spaces to the analogous problem for finite dimesional spaces. Then according to lemma 1 , we get that $r(\mu(z), A(z))=$ $\operatorname{dim} \operatorname{Ker} \tilde{A}(z)$ is semicontinuous.

\section{EXAMPLES}

Example 1. First, we introduce an approximate solution of the Cauchy problem for the Poisson equation $\triangle u=f$ near $D$ :

Let $\triangle$ be the standard Laplacian on $\mathbb{R}^{n}, D$ a compact subset in $\mathbb{R}^{n}$ with Lebesgue measure 0. Suppose that for any multi-index $\alpha$ with $|\alpha| \leq 1$ and any $j=1, \cdots, k$, a complex-valued Borel measure $\nu_{\alpha j}$ supported on $D$ is given. Instead of considering the Laplacian on $\mathbb{R}^{n}$, let us consider the Laplacian on the torus $\mathbb{R}^{n} / N \mathbb{Z}^{n}$ since a neighborhood of $D$ can be considered as an open set in the torus (if $N$ is large 
enough). From the local solvability theorem, we have that for any $f \in C^{\infty}(D)$ there exists $u \in C^{\infty}(D)$ such that $\triangle u=f$ and

$$
\sum_{|\alpha| \leq 1} \int_{D} u^{(\alpha)} d \nu_{\alpha j}=0, \quad j=1, \cdots, k .
$$

Since we only allow first order derivatives, actually the imposed conditions (1) are void. Obviously, the dimension of the solution space of $\Delta u=f$ is the same as the dimension of the solution space of $\triangle u=0$.

Consider the compact subset $D$ moved by elements of the orthogonal group $\mathbf{O}(n)$ or by translations. Since the Laplacian commutes with the above operations, the dimension of the space of solutions is constant.

Example 2. Let $X$ be a compact Riemann surface of genus $g$. Consider the operator

$$
A=\bar{\partial}: C^{\infty}(X) \rightarrow \Lambda^{0,1}(X) .
$$

Suppose that $k+l$ distinct points $x_{1}, \cdots, x_{k}, y_{1}, \cdots, y_{l}$ are given in $X$, and define

$$
D^{+}=\left\{x_{1}, \cdots, x_{k}\right\}, \quad D^{-}=\left\{y_{1}, \cdots, y_{l}\right\} .
$$

Define also in local real coordinates near the given points

$$
\begin{aligned}
& L^{+}=\left\{\sum_{i=1}^{k} c_{i} \delta\left(x-x_{i}\right), c_{i} \in \mathbb{C}\right\}, \\
& L^{-}=\left\{\sum_{j=1}^{l} c_{j} \delta\left(x-y_{j}\right), c_{j} \in \mathbb{C}\right\} .
\end{aligned}
$$

Let us consider the rigged divisor $\mu=\left(D^{+}, L^{+} ; D^{-}, L^{-}\right)$. Then the space $L(\mu, A)$ is the space of all meromorphic functions $f$ on $X$ which are allowed to have at most simple poles at $x_{1}, \cdots, x_{k}$ and are required to have zeros at $y_{1}, \cdots, y_{l}$. Note that

$$
A^{*}=\bar{\partial}: \Lambda^{1,0} \rightarrow \Lambda^{1,1}(X)=\Lambda^{2}(X) .
$$

Therefore $L\left(\mu^{-1}, A^{*}\right)$ is the space of all meromorphic $(1,0)$-forms which are allowed to have simple poles at $y_{1}, \cdots, y_{l}$ and are required to vanish at all the points $x_{1}, \cdots, x_{k}$.

The secondary spaces are as follows:

$$
\tilde{L}^{+}=\{0\}, \quad \tilde{L}^{-}=\{0\} .
$$

It follows that $\operatorname{deg}_{A}(\mu)=k-l$. Since ind $A=1-g$, the Gromov-Shubin theorem is reduced to the classical Riemman-Roch theorem and

$$
L(\mu, A)=H^{0}(X, \mathcal{O}(D))
$$

where $D=\sum x_{i}-\sum y_{j}$.

We know that any holomorphic automorphism of $X$ keeps $h^{0}(X, \mathcal{O}(D))$ constant. But for the case of genus $0, h^{0}(X, \mathcal{O}(D))$ is constant under any bijective map of $X$.

For the case of genus 1 , we identify the Riemann surface with $\mathbb{C} / \Lambda$. For a torus, we have a one-parameter family of holomorphic automorphisms, i.e., translations.

For the case of non-hyperelliptic and genus greater than 2, we identify the Riemann surface with its canonical curve. 
Since the canonical curve is intrinsically defined by a Riemann surface $X$, we want to interpret the Riemann-Roch theorem in terms of the geometry of the canonical curve:

Consider $D=\sum x_{i}$ on the Riemann surface. Then $r\left(\mu^{-1}, A^{*}\right)=h^{0}(X, \mathcal{K}(-D))$ is the number of hyperplanes containing the points $x_{i}$, and so $r(\mu, A)=h^{0}(X, \mathcal{O}(D))$ is the degree of $D$ minus the dimension of the linear space spanned by the points $x_{i}$ on the canonical curve. Hence, for a 'general' Riemann surface, we know where the dimension of $L(\mu, A)=H^{0}(X, \mathcal{O}(D))$ will jump.

Example 3. Let $X$ be a compact Riemann surface of genus 0 or 1 . Consider the operator

$$
A=\bar{\partial}: C^{\infty}(X) \rightarrow \Lambda^{0,1}(X) .
$$

Suppose that $k$ distinct points $x_{1}, \cdots, x_{k}$ are given in $X$ and define

$$
D^{+}=\left\{x_{1}, \cdots, x_{k}\right\} .
$$

Define also in local real coordinates near the given points

$$
L^{+}=\left\{\sum_{i=1}^{k} c_{i} \delta\left(x-x_{i}\right), c_{i} \in \mathbb{C}\right\} .
$$

Now, let us replace $D^{-}$and $L^{-}$of example 2 with the holomorphic moment conditions:

$$
\int_{\gamma} u z^{j} d z=0, \text { for } j=0, \cdots, l,
$$

where $z$ is a local coordinate and $\gamma$ is an arc in the same chart. Here, we assume that $\gamma$ does not contain $x_{i}$.

For the case of the Riemann sphere, consider the following space:

$$
\left\{u \in H^{0}\left(X, \mathcal{O}(D) \mid \int_{\gamma} u z^{j} d z=0, \text { for } j=0, \cdots, l\right\}\right.
$$

where $z$ is the coordinate of the finite plane $\mathbb{C}$. Then its dimension is constant under the affine transformation of $\mathbb{C}$ :

$$
w=a z+b, \quad \text { where } a, b \in \mathbb{C} .
$$

For the case of a torus $\mathbb{C} / \Lambda$, consider the following space:

$$
\left\{u \in H^{0}\left(X, \mathcal{O}(D) \mid \int_{\gamma} u z^{j} d z=0, \text { for } j=0, \cdots, l\right\},\right.
$$

where $z$ is the coordinate of the universal covering $\mathbb{C}$. Then its dimension is a constant under translations.

\section{Appendix}

Theorem 3. Suppose that $H$ and $H^{\prime}$ are two Hilbert spaces. Let $T(z): H \longrightarrow H^{\prime}$ be a continuous family of Fredholm operators parametrized by a variable $z$. Then for 
any fixed $z=z_{0}$, there exist a neighborhood $U$ and two finite dimensional subspaces $V \subset H$ and $V^{\prime} \subset H^{\prime}$ such that the following diagram commutes:

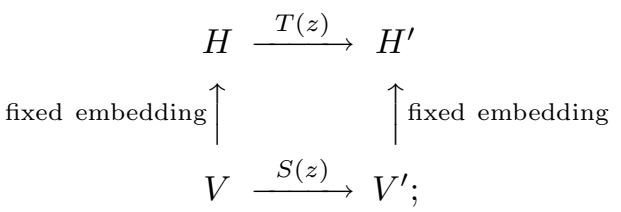

moreover, $\operatorname{dim} \operatorname{Ker} T(z)=\operatorname{dim} \operatorname{Ker} S(z)$ and $\operatorname{dim} \operatorname{Coker} T(z)=\operatorname{dim} \operatorname{Coker} S(z)$ for any $z \in U$.

Proof. Note that $\operatorname{codim} T\left(z_{0}\right) H$ is finite since $T\left(z_{0}\right)$ is a Fredholm operator. Therefore, $H^{\prime}$ can be decomposed into

$$
H^{\prime}=H_{0}{ }^{\prime} \oplus T\left(z_{0}\right) H,
$$

where $H_{0}{ }^{\prime} \perp T\left(z_{0}\right) H$.

Denote the projection map from $H^{\prime}$ to $H_{0}{ }^{\prime}$ by $P_{H_{0}}^{H^{\prime}}$. We can decompose $T(z)$ as

$$
T(z)=T_{1}(z) \oplus T_{2}(z)
$$

where $T_{1}(z)=P_{H_{0}}^{H^{\prime}} \circ T(z)$ and $T_{2}(z)=P_{T\left(z_{0}\right) H}^{H^{\prime}} \circ T(z)$. Since $T_{2}\left(z_{0}\right)$ is onto, there exists a neighborhood $U_{1}$ such that $T_{2}(z): H \longrightarrow T\left(z_{0}\right) H$ is onto for any $z \in U_{1}$.

On the other hand, we have

$$
H=\operatorname{Ker} T_{2}\left(z_{0}\right) \oplus\left(\operatorname{Ker} T_{2}\left(z_{0}\right)\right)^{\perp} .
$$

Denote by $i^{\perp}$ the embedding of $\left(\operatorname{Ker} T_{2}\left(z_{0}\right)\right)^{\perp}$. There exists a neighborhood $U \subset U_{1}$ such that $T_{2}(z) \circ i^{\perp}:\left(\operatorname{Ker} T_{2}\left(z_{0}\right)\right)^{\perp} \longrightarrow T\left(z_{0}\right) H$ is an isomorphism for any $z \in U$. Let $V$ be $\operatorname{Ker} T_{2}\left(z_{0}\right)$ and $V^{\prime}$ be $H_{0}{ }^{\prime}$. Let $S(z)$ be the composition of $T_{1}(z)$ and the embedding of $\operatorname{Ker} T_{2}\left(z_{0}\right)$. Then the theorem follows.

\section{REFERENCES}

[A-S] Atiyah, M. F., Singer, I. M.: The index of elliptic operators: III, Ann. of Math. (2) 87 (1968), 546-604. MR 38:5245

[G-H] Griffiths, P., Harris, J.: Principles of algebraic geometry, Wiley, New York, 1978. MR 80b: 14001

[G-S] Gromov, M., Shubin, M.A.: The Riemann-Roch theorem for elliptic operators and solvability of elliptic equations with additional conditions on compact subsets, Invent. Math. 117 (1994), 165-180. MR 95d:58121

[H1] Hörmander, L.: The analysis of linear partial differential operators, Vol. I, Springer, Berlin, Heidelberg, and New York, 1983. MR 85g:35002a

[H2] Hörmander, L.: The analysis of linear partial differential operators, Vol. III, Springer, Berlin, Heidelberg, and New York, 1985. MR 87d:35002a

Department of Mathematical Sciences, University of Durham, Science Laboratories, South Road, Durham, DH1 3LE, England

E-mail address: Lih-Chung.Wang@durham.ac.uk

Current address: Department of Mathematics, University of California, Riverside, California 92521

E-mail address: lwang@math.ucr.edu 